\title{
THE INFLUENCE OF SECOND ORDER NARROW-BAND COLORED NOISES ON NON-LINEAR RANDOM VIBRATIONS
}

\author{
NGUYEN DONG ANH, NGUYEN DUC TINH \\ Institute of Mechanics, NCST of Vietnam
}

\begin{abstract}
Since the effect of some nonlinear terms is lost during the first order averaging procedure, the higher order stochastic averaging method is developed to predict approximately the response of linear and lightly nonlinear systems subject to weakly external excitation of second order coloured noise random processes. Application to Duffing oscillator is considered.
\end{abstract}

\section{Introduction}

For many years the well-known averaging method, originally given by Krylov and Bogoliubov and then developed by Mitropolskii (Bogoliubov and Mitropolskii, 1961) has proved to be a very powerful approximate tool for investigating deterministic weakly nonlinear vibration problems. In the field of random vibration the averaging method was extended by Stratonovich (1963) and has a mathematically rigorous proof by Khasminskii (1963). At.present, the stochastic averaging method (SAM) is widely used in different problems of stochastic mechanics such as vibration, stability and reliability problems (see e.g. Ariaratnam \& Tam,1979; Bolotin; 1984; Ibrahim, 1985; Lin \& Cai, 1995; Roberts \& Spanos, 1986; Zhu, 1988).

It should be noted that principally only first order SAM has been applied in practice and usually to systems subject to white noise or wideband random processes. It is well-known, however, the effect of some non-linear terms is lost during the first order averaging procedure. In order to over come this insufficiency, different averaging procedures for obtaining approximate solutions have been developed (see e.g. Mitropolskii et al, 1992; Red-Horse \& Spanos, 1992; Sri Namachchivaya \& Lin, 1988; Zhu \& Lin, 1994; Zhu et al, 1997). Recently, a higher order averaging procedure using Fokker-Planck (FP) equation was developed in (Anh, 1993, 1995) and then applied to Van der Pol oscillator under white noise excitation (Anh \& Tinh, 1995 ). In the present paper this procedure is further developed to linear and lightly nonlinear systems subject to weakly external excitation of second order 
narrow-band coloured noise random processes.

\section{Narrow-band coloured noise random processes}

We consider a stationary coloured noise random process $c(t)$ which is the result of the passage of a white noise through the linear forming filter $L$ of order 2n:

$$
L(c(t))=\frac{d^{2 n} c(t)}{d t^{2 n}}+\sum_{j=0}^{2 n-1} \alpha_{j} \frac{d^{j} c(t)}{d t^{j}}=\sigma \dot{\xi}(t)
$$

where $\alpha_{j}, \sigma$ are constants, $\dot{\xi}(t)$ is a the zero-mean white noise process with unit intensity

$$
E(\dot{\xi}(t) \dot{\xi}(t+\tau))=\delta(\tau)
$$

where $E(\cdot)$ is the operator of expectation. It is supposed that all roots of the characteristic equation for the filter (2.1)

$$
\ell(\mu)=\mu^{2 n}+\sum_{j=0}^{2 n-1} \alpha_{j} \mu^{j}=0
$$

have negative real parts. The behaviour of $c(t)$ essentially depends on the roots of the characteristic equation (2.3) and one can get from (2.1) wideband or narrowband coloured noise processes, respectively.

\subsection{Narrow-band coloured noise}

Let the filter $L$ can be expressed in the form

$$
L(\cdot) \equiv L_{0}(\cdot)+\varepsilon L_{1}(\cdot)=\prod_{k=1}^{m}\left[\frac{d^{2}(\cdot)}{d t^{2}}+\omega_{k}^{2}(\cdot)\right]+\varepsilon L_{1}(\cdot)
$$

where $L_{1}$ is also a linear filter of order $q ; m, q \leq n ; \omega_{k}, k=1,2, \ldots, m$, are distinct positive values and $\omega_{k} \gg \varepsilon$. In this case it is supposed that one can get from (2.1), (2.4) a narrow- band coloured noise process.

\subsection{Second order narrow- band coloured noise} form

Coloured noise of second order $p(t)$ can be obtained by the filter $P$ of the

$$
P(p(t)) \equiv \ddot{p}(t)+2 \alpha \dot{p}(t)+\omega_{1}^{2} p(t)=2 \sigma_{2} \sqrt{\alpha} \omega_{1} \dot{\xi}(t)
$$


where $\alpha>0, \omega_{1}, \sigma_{2} \gg \varepsilon$. The auto-correlation function and the spectral density function of $p(t)$ are, respectively,

$$
\begin{aligned}
& R_{p}(\tau)=\sigma_{2}^{2} e^{-\alpha \tau}\left(\cos \sqrt{\omega_{1}^{2}-\alpha^{2}} \tau+\alpha\left(\omega_{1}^{2}-\alpha^{2}\right)^{-1}\left|\sin \sqrt{\omega_{1}^{2}-\alpha^{2}} \tau\right|\right) \\
& S_{p}(\omega)=\frac{2 \sigma_{2}^{2} \alpha \omega_{1}^{2}}{\pi\left[\left(\omega^{2}-\omega_{1}^{2}\right)^{2}+4 \alpha^{2} \omega^{2}\right]}
\end{aligned}
$$

The bandwidth of the process $p(t)$ is controlled by the value of the parameter $\alpha$. For the present analysis the coloured noise $p(t)$ is considered to be a wideband random process if $\alpha \gg \varepsilon$,. If $\alpha \sim \varepsilon$, i.e.

$$
\alpha=\varepsilon \alpha_{1}
$$

$p(t)$ is a narrow - band process and the filter (2.5) has the form (2.4), where

$$
P_{0}(\cdot)=\frac{d^{2}(\cdot)}{d t^{2}}+\omega_{1}^{2}(\cdot), \quad P_{1}(\cdot)=2 \alpha_{1} \frac{d(\cdot)}{d t}
$$

\section{Excitation of second order narrow-band coloured noise}

Consider a lightly damping system subject to external narrow-band second order coloured noise excitation

$$
\ddot{x}+\omega_{0}^{2} x=\varepsilon f_{1}(x, \dot{x})+\varepsilon^{2} f_{2}(x, \dot{x})+p(t)
$$

where $\omega_{0}$ is a positive constant, $\varepsilon>0$ is a small parameter, $p(t)$ is determined from (2.5) and (2.8), $f_{1}$ and $f_{2}$ are functions of $(x, \dot{x})$. In this case one gets $m=1$.

Eliminating $p(t)$ from (3.1), using (2.4), one gets

$$
\left[\frac{d^{2}(\cdot)}{d t^{2}}+\omega_{1}^{2}(\cdot)\right]\left(\ddot{x}+\omega_{0}^{2} x\right)=\varepsilon F_{1}+\varepsilon^{2} F_{2}+\varepsilon^{3}+\cdots+\sqrt{\varepsilon} \sigma_{1} \dot{\xi}(t)
$$

where it is denoted

$$
F_{1}=L_{0} f_{1}-L_{1}\left(\bar{x}+\omega_{0}^{2} x\right), \quad F_{2}=L_{0} f_{2}+L_{1} f_{1} .
$$

Suppose

$$
\left\|\omega_{0}-\omega_{\rho}\right\| \gg \varepsilon, \quad s, \rho=0,1, s \neq \rho .
$$

According to the averaging concept, a stationary solution of (3.2) is found in the form

$$
\frac{d^{i} x(t)}{d t^{i}}=a_{0} \omega_{0}^{i} \cos \left(\varphi_{0}+i \frac{\pi}{2}\right)+a_{1} \omega_{1}^{i} \cos \left(\varphi_{1}+i \frac{\pi}{2}\right), \quad i=0,1,2,3
$$


where as, $a_{s}, \varphi_{s}=0,1$ are new variables. By using Ito differentiation formula the equation (3.2) is transformed into the following system of equations [11]:

$$
\begin{aligned}
& \dot{a}_{s}=\varepsilon A_{1 s}(a, \varphi)+\varepsilon^{2} A_{2 s}(a, \varphi)+\varepsilon^{3}+\cdots-\sqrt{\varepsilon} \sigma_{1} \frac{\sin \varphi_{s}}{\omega_{s} \Omega_{s}} \dot{\xi}(t) \\
& \dot{\varphi}_{s}=\omega_{s}+\varepsilon B_{1 s}(a, \varphi)+\varepsilon^{2} B_{2 s}(a, \varphi)+\varepsilon^{3}+\cdots-\sqrt{\varepsilon} \sigma_{i} \frac{\cos \varphi_{s}}{a_{s} \omega_{s} \Omega_{s}} \dot{\xi}(t)
\end{aligned}
$$

where it is denoted

$$
\begin{aligned}
& A_{1 s}(a, \varphi)=\left\{-\frac{F_{1}(a, \varphi)}{\omega_{s} \Omega_{s}} \sin \varphi_{s}+\frac{\sigma_{1}^{2} \cos ^{2} \varphi_{s}}{2 a_{s} \omega_{s} \Omega_{s}^{2}}\right\} \\
& B_{1 s}(a, \varphi)=\left\{-\frac{F_{1}(a, \varphi)}{\omega_{s} \Omega_{s} a_{s}} \cos \varphi_{s}-\frac{\sigma_{1}^{2} \sin \varphi_{s} \cos \varphi_{s}}{\omega_{s}^{2} \Omega_{s}^{2} a_{s}^{2}}\right\} \\
& A_{2 s}(a, \varphi)=-\frac{F_{2}(a, \varphi)}{\omega_{s} \Omega_{s} a_{s}} \cos \varphi_{s} \\
& B_{2 s}(a, \varphi)=-\frac{F_{2}(a, \varphi)}{\omega_{s} \Omega_{s} a_{s}} \cos \varphi_{s} \\
& \sigma_{1}=2 \sigma_{2} \sqrt{\alpha_{1}} \omega_{1} \\
& \Omega_{0}=-\Omega_{1}=\omega_{1}^{2}-\omega_{0}^{2}, \quad a=\left(a_{0}, a_{1}\right), \quad \varphi=\left(\varphi_{0}, \varphi_{1}\right) \\
& F_{1}=\ddot{f}_{1}+\omega_{1}^{2} f_{1}-2 \alpha_{1}\left(\dddot{x}+\omega_{0}^{2} \dot{x}\right) \\
& F_{2}=\ddot{f}_{2}+\omega_{1}^{2} f_{2}+2 \alpha_{1} \dot{f}_{1}
\end{aligned}
$$

in which

$$
x^{(i)}(t)=\sum_{\rho=0}^{1} a_{\rho} \omega_{\rho}^{i} \cos \left(\varphi_{\rho}+i \frac{\pi}{2}\right), \quad i=0,1,2,3 .
$$

The Fokker-Planck equation for the stationary probability density function $W(a, \varphi)$ corresponding to the system (3.6) takes form

$$
\sum_{s=0}^{1} \omega_{s} \frac{\partial W}{\partial \varphi_{s}}=-\varepsilon\left[A_{1}, B_{1}\right] L(W)-\varepsilon^{2}\left[A_{2}, B_{2}\right] L(W)+\varepsilon^{3} \ldots
$$

where the operators $\left[A_{v}, B_{v}\right] L(\cdot), v=1,2$, are defined as follows

$$
\begin{aligned}
& {\left[A_{1}, B_{1}\right] L(W)=}\left.\sum_{s=0}^{1}\left[\frac{\partial}{\partial a_{s}}\left(A_{1 s} W\right)+\frac{\partial}{\partial \varphi_{s}} W\right)\right]- \\
&-\frac{\sigma_{1}^{2}}{2} \sum_{s=0}^{1} \sum_{\rho=0}^{1}\left\{\frac{\partial^{2}}{\partial a_{s} \partial a_{\rho}}\left(\frac{\sin \varphi_{s} \sin \varphi_{\rho}}{\omega_{s} \omega_{\rho} \Omega_{s} \omega_{\rho}} W\right)+\frac{\partial^{2}}{\partial a_{s} \partial \varphi_{\rho}}\left(\frac{\sin \varphi_{s} \cos \varphi_{\rho}}{a_{\rho} \omega_{s} \omega_{\rho} \Omega_{s} \Omega_{\rho}} W\right)+\right. \\
&\left.+\frac{\partial^{2}}{\partial \varphi_{s} \partial \varphi_{\rho}}\left(\frac{\cos \varphi_{s} \cos \varphi_{\rho}}{a_{s} a_{\rho} \omega_{s} \omega_{p} \Omega_{s} \Omega_{\rho}} W\right)\right\} \\
& {\left[A_{2}, B_{2}\right] L(W)=} \sum_{s=0}^{1}\left[\frac{\partial}{\partial a_{s}}\left(A_{2}, W\right)+\frac{\partial}{\partial \varphi_{s}}\left(B_{2}, W\right)\right]
\end{aligned}
$$


where as, $a_{s}, \varphi_{s}=0,1$ are new variables. By using Ito differentiation formula the equation (3.2) is transformed into the following system of equations [11]:

$$
\begin{aligned}
& \dot{a}_{s}=\varepsilon A_{1 s}(a, \varphi)+\varepsilon^{2} A_{2 s}(a, \varphi)+\varepsilon^{3}+\cdots-\sqrt{\varepsilon} \sigma_{1} \frac{\sin \varphi_{s}}{\omega_{s} \Omega_{s}} \dot{\xi}(t) \\
& \dot{\varphi}_{s}=\omega_{s}+\varepsilon B_{1 s}(a, \varphi)+\varepsilon^{2} B_{2 s}(a, \varphi)+\varepsilon^{3}+\cdots-\sqrt{\varepsilon} \sigma_{i} \frac{\cos \varphi_{s}}{a_{s} \omega_{s} \Omega_{s}} \dot{\xi}(t)
\end{aligned}
$$

where it is denoted

$$
\begin{aligned}
& A_{1 s}(a, \varphi)=\left\{-\frac{F_{1}(a, \varphi)}{\omega_{s} \Omega_{s}} \sin \varphi_{s}+\frac{\sigma_{1}^{2} \cos ^{2} \varphi_{s}}{2 a_{s} \omega_{s} \Omega_{s}^{2}}\right\} \\
& B_{1 s}(a, \varphi)=\left\{-\frac{F_{1}(a, \varphi)}{\omega_{s} \Omega_{s} a_{s}} \cos \varphi_{s}-\frac{\sigma_{1}^{2} \sin \varphi_{s} \cos \varphi_{s}}{\omega_{s}^{2} \Omega_{s}^{2} a_{s}^{2}}\right\} \\
& A_{2 s}(a, \varphi)=-\frac{F_{2}(a, \varphi)}{\omega_{s} \Omega_{s} a_{s}} \cos \varphi_{s} \\
& B_{2 s}(a, \varphi)=-\frac{F_{2}(a, \varphi)}{\omega_{s} \Omega_{s} a_{s}} \cos \varphi_{s} \\
& \sigma_{1}=2 \sigma_{2} \sqrt{\alpha_{1}} \omega_{1} \\
& \Omega_{0}=-\Omega_{1}=\omega_{1}^{2}-\omega_{0}^{2}, \quad a=\left(a_{0}, a_{1}\right), \quad \varphi=\left(\varphi_{0}, \varphi_{1}\right) \\
& F_{1}=\ddot{f}_{1}+\omega_{1}^{2} f_{1}-2 \alpha_{1}\left(\ddot{x}+\omega_{0}^{2} \dot{x}\right) \\
& F_{2}=\ddot{f}_{2}+\omega_{1}^{2} f_{2}+2 \alpha_{1} \dot{f}_{1}
\end{aligned}
$$

in which

$$
x^{(i)}(t)=\sum_{\rho=0}^{1} a_{\rho} \omega_{\rho}^{i} \cos \left(\varphi_{\rho}+i \frac{\pi}{2}\right), \quad i=0,1,2,3 .
$$

The Fokker-Planck equation for the stationary probability density function $W(a, \varphi)$ corresponding to the system (3.6) takes form

$$
\sum_{s=0}^{1} \omega_{s} \frac{\partial W}{\partial \varphi_{s}}=-\varepsilon\left[A_{1}, B_{1}\right] L(W)-\varepsilon^{2}\left[A_{2}, B_{2}\right] L(W)+\varepsilon^{3} \ldots
$$

where the operators $\left[A_{v}, B_{v}\right] L(\cdot), v=1,2$, are defined as follows

$$
\begin{aligned}
& {\left[A_{1}, B_{1}\right] L(W)=}\left.\sum_{s=0}^{1}\left[\frac{\partial}{\partial a_{s}}\left(A_{1 s} W\right)+\frac{\partial}{\partial \varphi_{s}} W\right)\right]- \\
&-\frac{\sigma_{1}^{2}}{2} \sum_{s=0}^{1} \sum_{\rho=0}^{1}\left\{\frac{\partial^{2}}{\partial a_{s} \partial a_{\rho}}\left(\frac{\sin \varphi_{s} \sin \varphi_{\rho}}{\omega_{s} \omega_{\rho} \Omega_{s} \omega_{\rho}} W\right)+\frac{\partial^{2}}{\partial a_{s} \partial \varphi_{\rho}}\left(\frac{\sin \varphi_{s} \cos \varphi_{\rho}}{a_{\rho} \omega_{s} \omega_{\rho} \Omega_{s} \Omega_{\rho}} W\right)+\right. \\
&\left.+\frac{\partial^{2}}{\partial \varphi_{s} \partial \varphi_{\rho}}\left(\frac{\cos \varphi_{s} \cos \varphi_{\rho}}{a_{s} a_{\rho} \omega_{s} \omega_{p} \Omega_{s} \Omega_{\rho}} W\right)\right\} \\
& {\left[A_{2}, B_{2}\right] L(W)=\sum_{s=0}^{1}\left[\frac{\partial}{\partial a_{s}}\left(A_{2}, W\right)+\frac{\partial}{\partial \varphi_{s}}\left(B_{2}, W\right)\right] }
\end{aligned}
$$


It can be shown that the operators $\left[A_{v}, B_{v}\right] L(\cdot)$ are linear ones. We seek then the solution of (3.9) in the form

$$
W(a, \varphi)=W_{0}(a, \varphi)+\varepsilon W_{1}(a, \varphi)+\varepsilon^{2} W_{2}(a, \varphi)+\ldots
$$

Substituting (3.11) into (3.9) yields

$$
\begin{gathered}
\sum_{s=0}^{1} \omega_{s}\left[\frac{\partial W_{0}}{\partial \varphi_{s}}+\varepsilon \frac{\partial W_{1}}{\partial \varphi_{s}}+\varepsilon^{2} \frac{\partial W_{2}}{\partial \varphi_{s}}+\ldots\right]=-\varepsilon\left[A_{1}, B_{1}\right] L\left(W_{0}\right)- \\
-\varepsilon^{2}\left\{\left[A_{2}, B_{2}\right] L\left(W_{0}\right)+\left[A_{1}, B_{1}\right] L\left(W_{1}\right)\right\}+\ldots
\end{gathered}
$$

Comparing the coefficients of like powers of $\varepsilon$ one obtains

$$
\begin{aligned}
\varepsilon^{0}: & \sum_{s=0}^{1} \omega_{s} \frac{\partial W_{0}}{\partial \varphi_{s}}=0 \\
\varepsilon^{1}: & \sum_{s=0}^{1} \omega_{s} \frac{\partial W_{1}}{\partial \varphi_{s}}=-\left[A_{1}, B_{1}\right] L\left(W_{0}\right) \\
\varepsilon^{2}: & \sum_{s=0}^{1} \omega_{s} \frac{\partial W_{2}}{\partial \varphi_{s}}=-\left\{\left[A_{2}, B_{2}\right] L\left(W_{0}\right)+\left[A_{1}, B_{1}\right] L\left(W_{1}\right)\right\}
\end{aligned}
$$

From (3.13) it gives a periodic solution with respect to $\varphi$ as follows

$$
W_{0}=W_{0}(a) .
$$

Substituting (3.16) into (3.14) yields

$$
\sum_{s=0}^{1} \omega_{s} \frac{\partial W_{1}}{\varphi_{s}}=-\left[A_{1}, B_{1}\right] L\left(W_{0}(a)\right) .
$$

The arbitrary integration function $W_{0}(a)$ must be chosen from the condition for the function $W_{1}(a, \varphi)$ to be periodic with respect to $\varphi$. Thus, one gets from (3.17)

$$
\left\langle\left[\left[A_{1}, B_{1}\right] L\left(W_{0}(a)\right)\right]\right\rangle=0
$$

where $\langle\cdot\rangle$ is the averaging operator with respect to phase $\varphi$

$$
\langle\cdot\rangle=\frac{1}{(2 \pi)^{2}} \int_{0}^{2 \pi} \int_{0}^{2 \pi} \cdot(\cdot) d \varphi_{0} d \varphi_{1}
$$


Substituting (3.10) into (3.18) yields

$$
\sum_{s=0}^{1}\left\{\frac{\partial}{\partial a}\left(\left\langle A_{1 s}\right\rangle W_{0}(a)\right)-\frac{\sigma_{1}^{2}}{4 \omega_{s}^{2} \Omega_{s}^{2}} \frac{\partial^{2} W_{0}(a)}{\partial a_{s}^{2}}\right\}=0
$$

The averaged Fokker - Planck equation (3.20) can be considered as an extension into the case of narrow - band excitation of the well - known first order averaged FP equation obtained for the white noise excitation. Further, it is seen that the averaged FP equation (3.20) is obtained by equating zero the averaged value of the coefficient of power $\varepsilon$ in the original FP equation (3.9).

The second term $W_{1}(a, \varphi)$ in (3.11) is determined from (3.14), using Fourier expansion

$$
\left[A_{1}, B_{1}\right] L\left(W_{0}(a)\right)=W_{0}(a) \sum_{k_{0}} \sum_{k_{1}} C_{k_{0} k_{1}}(a) e^{i\left(k_{0} \varphi_{0}+k_{1} \varphi_{1}\right)}
$$

where

$$
C_{k_{0} k_{1}}(a)=\frac{1}{W_{0}(a)(2 \pi)^{2}} \int_{0}^{2 \pi} \int_{0}^{2 \pi}\left[A_{1}, B_{1}\right] L\left(W_{0}(a) \cdot e^{-i\left(k_{0} \varphi_{0}+k_{1} \varphi_{1}\right)} d \varphi_{0} d \varphi_{1} .\right.
$$

Substituting (3.21) into (3.14) yields

$$
W_{1}(a, \varphi)=W_{0}(a)\left[W_{10}(a)+\sum_{k_{0}} \sum_{k_{1}} \frac{C_{k_{0} k_{1}}}{k_{0} \varphi_{0}+k_{1} \varphi_{1}} e^{i\left(k_{0} \varphi_{0}+k_{1} \varphi_{1}\right)}\right]
$$

where

$$
k_{0} \omega_{0}+k_{1} \omega_{1} \neq 0
$$

The arbitrary integration function $W_{10}(a)$ must be chosen from the condition for the function $W_{2}(a, \varphi)$ to be periodic. Analogously, one can find third term $W_{2}(a, \varphi)$ in (3.11).

\section{Application}

In order to illustrate the procedure proposed one considers the Duffing system whose equation of motion takes the form:

$$
\ddot{x}+\omega^{2} x=-2 \varepsilon h \dot{x}-\varepsilon^{2} \gamma x^{3}+p(t)
$$


Substituting (3.10) into (3.18) yields

$$
\sum_{s=0}^{1}\left\{\frac{\partial}{\partial a}\left(\left\langle A_{1 s}\right\rangle W_{0}(a)\right)-\frac{\sigma_{1}^{2}}{4 \omega_{s}^{2} \Omega_{s}^{2}} \frac{\partial^{2} W_{0}(a)}{\partial a_{s}^{2}}\right\}=0 .
$$

The averaged Fokker - Planck equation (3.20) can be considered as an extension into the case of narrow - band excitation of the well - known first order averaged FP equation obtained for the white noise excitation. Further, it is seen that the averaged FP equation (3.20) is obtained by equating zero the averaged value of the coefficient of power $\varepsilon$ in the original FP equation (3.9).

The second term $W_{1}(a, \varphi)$ in (3.11) is determined from (3.14), using Fourier expansion

$$
\left[A_{1}, B_{1}\right] L\left(W_{0}(a)\right)=W_{0}(a) \sum_{k_{0}} \sum_{k_{1}} C_{k_{0} k_{1}}(a) e^{i\left(k_{0} \varphi_{0}+k_{1} \varphi_{1}\right)}
$$

where

$$
C_{k_{0} k_{1}}(a)=\frac{1}{W_{0}(a)(2 \pi)^{2}} \int_{0}^{2 \pi} \int_{0}^{2 \pi}\left[A_{1}, B_{1}\right] L\left(W_{0}(a) \cdots e^{-i\left(k_{0} \varphi_{0}+k_{1} \varphi_{1}\right)} d \varphi_{0} d \varphi_{1} .\right.
$$

Substituting (3.21) into (3.14) yields

$$
W_{1}(a, \varphi)=W_{0}(a)\left[W_{10}(a)+\sum_{k_{0}} \sum_{k_{1}} \frac{C_{k_{0} k_{1}}}{k_{0} \varphi_{0}+k_{1} \varphi_{1}} e^{i\left(k_{0} \varphi_{0}+k_{1} \varphi_{1}\right)}\right]
$$

where

$$
k_{0} \omega_{0}+k_{1} \omega_{1} \neq 0
$$

The arbitrary integration function $W_{10}(a)$ must be chosen from the condition for the function $W_{2}(a, \varphi)$ to be periodic. Analogously, one can find third term $W_{2}(a, \varphi)$ in (3.11).

\section{Application}

In order to illustrate the procedure proposed one considers the Duffing system whose equation of motion takes the form:

$$
\ddot{x}+\omega^{2} x=-2 \varepsilon h \dot{x}-\epsilon^{2} \gamma x^{3}+p(t)
$$


where $h, \gamma>0, p(t)$ is a narrow - band random process obtained by the filter (2.5). In this case one gets:

$$
f_{1}(x, \dot{x})=-2 h \dot{x}, \quad f_{2}(x, \dot{x})=-\gamma x^{3} .
$$

Substituting (4.2) into (3.7) yields:

$$
\left\{\begin{array}{l}
F_{1}=-2\left(\alpha_{1}+h\right) \dddot{x}-2\left(\alpha_{1} \omega_{0}^{2}+h \omega_{1}^{2}\right) \dot{x} \\
F_{2}=-2 h \alpha_{1} \ddot{x}-\gamma \omega_{1}^{2} x^{3}-3 \gamma\left(x^{2} \ddot{x}+x \dot{x}^{2}\right) .
\end{array}\right.
$$

Using (2.10), $(3,4)$ and $(4.3)$ for equation (2.24) one gets:

$$
W_{0}(a)=C a_{0} a_{1} \exp \left\{-\frac{\left(\omega_{1}^{2}-\omega_{0}^{2}\right)^{2}}{2 \sigma_{2}^{2}}\left(a_{1}^{2}+\frac{h \omega_{0}^{2}}{\alpha_{1} \omega_{1}^{2}} a_{0}^{2}\right)\right\}
$$

( $C=$ const). This solution is the same as the result obtained by using the classical SAM. Substituting (4.4) into (3.14) and using (3.7), (3.8) and (4.3) after some calculations one obtains:

$$
\begin{aligned}
& W_{11}(a, \varphi)= \\
& =-\frac{h}{\omega_{0}} \sin ^{2} \varphi_{0}-\frac{\alpha_{1}}{\omega_{1}} \sin ^{2} \varphi_{1}+\left(\frac{2 h \omega_{0} \Omega_{0}}{\sigma_{2}^{2}} a_{0} a_{1}-\frac{4 \alpha_{1} \omega_{1}^{2} \sigma_{2}^{2}}{\omega_{0} \Omega_{0}^{3}} \frac{1}{a_{0} a_{1}}\right) \sin \varphi_{0} \cos \varphi_{1} \\
& \quad-\left(\frac{2 h \omega_{0}^{2} \Omega_{0}}{\sigma_{2}^{2} \omega_{1}} a_{0} a_{1}-\frac{4 \alpha_{1} \omega_{1} \sigma_{2}^{2}}{\omega_{0}^{3}} \frac{1}{a_{0} a_{1}}\right) \cos \varphi_{0} \sin \varphi_{1} .
\end{aligned}
$$

Substituting (4.4) and (4.5) into (3.15) one gets the equation for the arbitrary function $W_{10}(a)$ in the form:

$$
\begin{aligned}
& \sum_{s=0}^{1}\left\{\frac{\partial}{\partial a_{s}}\left[\left\langle A_{1 s}\right\rangle W_{0}(a) W_{10}(a)\right]-\frac{\sigma_{1}^{2}}{4 \omega_{s}^{2} \Omega_{s}^{2}} \frac{\partial^{2}}{\partial a_{s}^{2}}\left[W_{0}(a) W_{10}(a)\right]\right\}= \\
& =-\sum_{s=0}^{1} \frac{\partial}{\partial a_{s}}\left[\left\langle A_{2 s}\right\rangle W_{0}(a)\right]=\frac{3 \gamma}{4}\left[\frac{\partial}{\partial a_{0}}\left(\frac{\omega_{0}}{\Omega_{0}} a_{0}^{3} W_{0}\right)+\frac{\partial}{\partial a_{1}}\left(\frac{\omega_{1}}{\Omega_{1}} a_{1}^{3} W_{0}\right)\right] .
\end{aligned}
$$

From (4.6) one gets:

$$
W_{10}(a)=-\frac{3 \gamma}{4 \sigma_{1}^{2}}\left(\omega_{0}^{3} \Omega_{0} a_{0}^{4}+\omega_{1}^{3} \Omega_{1} a_{1}^{4}\right)
$$

Thus, the second order apprpximate solution of the FP equation (3.9) for the Duffing system (4.1) takes the form:

$$
W(a, \varphi)=W_{0}(a)\left\{1+\varepsilon\left[W_{10}(a)+W_{11}(a)\right]\right\}
$$


where $W_{0}(a), W_{11}(a, \varphi)$ and $W_{10}(a)$ are defined in (4.4), (4.5) and (4.7) respectively. It is seen from (4.4) and (4.8) that the solution $W(a, \varphi)$ in (4.8) is different from the solution obtained by using the classical SAM and the effect of the non linear term $\varepsilon^{2} \gamma x^{3}$ is shown in (4.8).

The corresponding approximate mean square $E\left[x^{2}\right]$ is to be found

$$
E\left[x^{2}\right]=\int_{0}^{2 \pi} \int_{0}^{2 \pi} \int_{0}^{\infty} \int_{0}^{\infty} x^{2} W(a, \varphi) d \varphi_{0} d \varphi_{1} d a_{0} d a_{1} .
$$

Substituting (4.8) and $x=a_{0} \cos \varphi_{0}+a_{1} \cos \varphi_{1}$ into (4.9), after calculations one obtains:

$$
\begin{aligned}
& E\left(x^{2}\right)= \\
& =\frac{\int_{0}^{\infty} \int_{0}^{\infty}\left(a_{0}^{2}+a_{1}^{2}\right)\left[1+\varepsilon W_{10}(a)\right] a_{0} a_{1} \exp \left\{-\frac{\left(\omega_{1}^{2}-\omega_{0}^{2}\right)^{2}}{2 \sigma_{2}^{2}}\left(a_{1}^{2}+\frac{h \omega_{0}^{2}}{\alpha_{1} \omega_{1}^{2}} a_{0}^{2}\right)\right\} d a_{0} d a_{1}}{2 \int_{0}^{\infty} \int_{0}^{\infty}\left[1+\varepsilon W_{10}(a)\right] a_{0} a_{1} \exp \left\{-\frac{\left(\omega_{1}^{2}-\omega_{0}^{2}\right)^{2}}{2 \sigma_{2}^{2}}\left(a_{1}^{2}+\frac{h \omega_{0}^{2}}{\alpha_{1} \omega_{1}^{2}} a_{0}^{2}\right)\right\} d a_{0} d a_{1}} \\
& =\frac{\sigma_{2}^{2}\left(h \omega_{0}^{2}+\alpha_{1} \omega_{1}^{2}\right)}{h \omega_{0}^{2}\left(\omega_{1}^{2}-\omega_{0}^{2}\right)^{2}}-6 \varepsilon \gamma \omega_{1} \sigma_{2}^{4}\left(h^{3} \omega_{0}^{3}+\alpha_{1}^{3} \omega_{1}^{3}\right)+\varepsilon^{2} \ldots
\end{aligned}
$$

In the case $\gamma=0$ (linear system) one gets:

$$
E\left[x^{2}\right]=\frac{\sigma_{2}^{2}\left(h \omega_{0}^{2}+\alpha_{1} \omega_{1}^{2}\right)}{h \omega_{0}^{2}\left(\omega_{1}^{2}-\omega_{0}^{2}\right)^{2}} .
$$

It is seen from (4.10) and (4.11) that in the case of Duffing system the mean square $E\left[x^{2}\right]$ reduces in comparison with the linear case.

\section{Conclusion}

For many years the stochastic averaging method has been a very useful tool for investigating non-linear random vibration systems. However, the effect of some non-linear terms cannot be investigated by using the classical first order SAM. In this paper, the higher order stochastic averaging method is developed to predict approximately the response of linear and lightly nonlinear systems subject to weakly external excitation of second order narrow-band coloured noise random processes. 
Acknowledgment. Support from the Council for natural sciences of Vietnam is gratefully acknowledged.

\section{REFERENCES}

1. Anh N. D. Higher order approximate solutions in stochastic averaging method. In Proc. of NCSR of VN, 5 (1993), 19-26.

2. Anh N. D. Higher order averaging method of coefficients in Fokker-Planck equation. In special volume: Advances in nonlinear structural dynamics of SADHANA, Indian Acad. of Sc., (1995) 373-388.

3. Anh N. D. and Tinh N. D. Higher order averaging solutions for Van der Pol oscillator. In Proc. Int. Conf. On Nonlinear Stochastic Dynamics, Hanoi, Viet nam, (1995) 27-38.

4. Ariaratnam S. T., Tam D. S. F. Random vibration and stability of a linear parametrically excited oscillator. Z. Angew. Math. Mech., 59, No 2, (1979), 79-84.

5. Bogoliubov N. N. and Mitropolskii Iu. A. Asymptotic methods in the theory of nonlinear oscillations, New York: Gordon and Breach (1961).

6. Bolotin V. V. Random vibration of elastic systems. Hague: Martinus Nijhoff (1984).

7. Ibrahim R. A. Parametric random vibration (Hertfordshire New York: Research Studies Press/John Wiley and Sons) (1985).

8. Iyengar R. N. Approximate analysis of nonlinear systems under narrow band random excitation, In Proc. of IUTAM Sympo-sium on Nonlinear Stochastic Mechanics, Turin, Italy, (1992), 309-320.

9. Khasminskii R. Averaging principle for the parabolic and elliptic differential equations and for Markov processes with small diffusion. Theor. Prob. Appl., 8, (1963), 1- 21 (in Russian).

10. Lin Y. K, Cai G. Q. Probabilistic structural dynamics, Mc Graw-Hill, Inc (1995).

11. Mitropolskii Iu. A., Dao, N. V, Anh, N. D. Nonlinear oscillations in systems of arbitrary order, Naukova - Dumka, Kiev, (1992) (in Russian):

12. Red-Horse J. R. Spanos P. D. A generalization to stochastic averaging in random vibration. Int. J. Nonlinear Mech. 27 (1992), 85-101.

13. Roberts J. B, Spanos P. T. D. Stochastic averaging: An approximate method 
of solving random vibration problem. Int. J. Nonlinear Mech. 21 (1986), 111-134.

14. Sri Namachchivaya N., and Lin Y. K. Application of stochastic averaging for nonlinear systems with high damping, Probabilistic Engineering Mechanics, 3, No 3, (1988), 159-167.

15. Stratonovich R. L. Topics in the theory of a random noise New York: Gordon and Breach Vol. 1 (1963).

16. Zhu W. Q. Stochastic averaging method in random vibrations. Appl. Mech. Rev. 41 (1988), 189-199.

17. Zhu W. Q., Yu, M. Q, Lin Y. K. On improved stochastic averaging procedure. Probab. Eng. Mech. 9 (1994), 203-212.

18. Zhu W. Q. et al., Stochastic averaging of quasi-integrable Hamiltonian systems, J. Applied Mech., 64 (1997), 975-984.

Received March 20, 1999

\section{ẢNH HƯớNG CỬA Kf́CH ĐÔ̂NG ÔN MÀU DẢI HEP BÂC HAI LÊN DAO ĐộNG NGẪU NHIÊN PHI TUYẾN}

Phương pháp trung bình ngẫu nhiên bậc nhất kinh điển đã được áp dụng rộng rãi đối với các hệ cơ học phi tuyến. Tuy nhiên, hiệu ứng của nhiều số hạng phi tuyến không được thể hiện khi sử dụng phương pháp này. Để khắc phục nhược điểm trên, phương pháp trung bình ngẫu nhiên bậc cao đã được phát triển đối với các hệ cơ học phi tuyến chịu kích động ngẫu nhiên dạng ồn trăng. Trong bài báo này, phương pháp tiếp tục được trình bày đối với các hệ phi tuyến yếu chịu kích động ngẫu nhiên dạng ồn màu dải hẹp bậc hai. Sau đó phương pháp được áp dụng để xác định nghiệm xấp xỉ bậc hai cưa phương trình Fokker - Planck đối với hệ dao động dạng Duffing.

Institute of Mechanics

264 Doi Can Street, Hanoi, Vietnam 